DISTRIBUTION STATEMENT A. Approved for public release; distribution is unlimited.

\title{
X-band Observations of Waves, Algorithm Development, and Validation High Resolution Wave-Air-Sea Interaction DRI
}

\author{
Eric J. Terrill \\ Scripps Institution of Oceanography \\ mail code 0213 \\ La Jolla, CA 92093-0213
}

phone: (858) 822-3101 fax (858) 534-7132 email: et@mpl.ucsd.edu

Award \#: N00014-07-1-0216

\section{LONG-TERM GOALS}

Our goal is to better understand physics of the air-sea interface including ocean wave generation, wave propagation, and air-sea fluxes.

\section{OBJECTIVES}

The potential to generate deterministic descriptions of the ocean's surface at elevated sea states using both modern observational techniques and computationally intensive, nonlinear wave models now exists. Investments in research to successfully validate and improve these techniques and models will improve the planning and execution of at-sea naval operations and further our understanding of air-sea interaction processes.

While signatures of the sea surface (sea clutter) are visible in the near range $(<3 \mathrm{~nm})$ of any nautical $\mathrm{X}$-band radar image, this information is generally filtered by the radar due to its design for navigation purposes. The sea clutter is a result of the backscatter at X-band principally composed of the Bragg scatter from $\mathrm{O}(1) \mathrm{cm}$ scale capillary-gravity waves which are modulated by the underlying long ocean waves. As a result, sea clutter is a rich signal which contains information about the 2-D structure of ocean waves. Successful measurement and interpretation of the clutter may lead to an ability to reconstruct ocean wave heights over the domain observed by the radar. The objectives of this program are to research our ability to generate phase-resolved maps of the ocean sea surface. We also have the secondary objectives to use radar to retrieve environmental variables including currents, winds, Langmuir cells, and breaking waves.

\section{APPROACH}

We have successfully completed our $5^{\text {th }}$ year of the 5 year Hi-Resolution (HIRES) Defense Research Initiative (DRI). Our experimental approach for the program was a series of field programs designed to gather data, test new instruments and algorithms, and the synthesis of data. Our primary system for collecting X-band radar backscatter is a radar acquisition system interfaced to a Furuno marine radar. We selected a commercially available system called Wave and Surface Current Monitoring System (WAMOS) that can be configured for both raw backscatter data acquisition and realtime processing of radar data for bulk wave parameters (non-phase resolved). For our research, the system is primarily 


\section{Report Documentation Page}

Form Approved

OMB No. 0704-0188

Public reporting burden for the collection of information is estimated to average 1 hour per response, including the time for reviewing instructions, searching existing data sources, gathering and maintaining the data needed, and completing and reviewing the collection of information. Send comments regarding this burden estimate or any other aspect of this collection of information,

including suggestions for reducing this burden, to Washington Headquarters Services, Directorate for Information Operations and Reports, 1215 Jefferson Davis Highway, Suite 1204, Arlington

VA 22202-4302. Respondents should be aware that notwithstanding any other provision of law, no person shall be subject to a penalty for failing to comply with a collection of information if it

does not display a currently valid OMB control number.

\begin{tabular}{|c|c|c|}
\hline $\begin{array}{l}\text { 1. REPORT DATE } \\
\mathbf{2 0 1 2}\end{array}$ & $\begin{array}{l}\text { 2. REPORT TYPE } \\
\text { N/A }\end{array}$ & $\begin{array}{l}\text { 3. DATES COVERED } \\
\text { - }\end{array}$ \\
\hline \multirow{3}{*}{\multicolumn{2}{|c|}{$\begin{array}{l}\text { 4-band Observations of Waves, Algorithm Development, and Validation } \\
\text { High Resolution Wave-Air-Sea Interaction DRI }\end{array}$}} & 5a. CONTRACT NUMBER \\
\hline & & 5b. GRANT NUMBER \\
\hline & & 5c. PROGRAM ELEMENT NUMBER \\
\hline \multirow{3}{*}{\multicolumn{2}{|c|}{ 6. AUTHOR(S) }} & 5d. PROJECT NUMBER \\
\hline & & 5e. TASK NUMBER \\
\hline & & 5f. WORK UNIT NUMBER \\
\hline \multicolumn{2}{|c|}{$\begin{array}{l}\text { 7. PERFORMING ORGANIZATION NAME(S) AND ADDRESS(ES) } \\
\text { Scripps Institution of Oceanography mail code } 0213 \text { La Jolla, CA } \\
\text { 92093-0213 }\end{array}$} & $\begin{array}{l}\text { 8. PERFORMING ORGANIZATION } \\
\text { REPORT NUMBER }\end{array}$ \\
\hline \multirow{2}{*}{\multicolumn{2}{|c|}{ 9. SPONSORING/MONITORING AGENCY NAME(S) AND ADDRESS(ES) }} & 10. SPONSOR/MONITOR'S ACRONYM(S) \\
\hline & & $\begin{array}{l}\text { 11. SPONSOR/MONITOR'S REPORT } \\
\text { NUMBER(S) }\end{array}$ \\
\hline
\end{tabular}

12. DISTRIBUTION/AVAILABILITY STATEMENT

Approved for public release, distribution unlimited

13. SUPPLEMENTARY NOTES

The original document contains color images.

14. ABSTRACT

15. SUBJECT TERMS

16. SECURITY CLASSIFICATION OF:

a. REPORT

unclassified b. ABSTRACT

unclassified
17. LIMITATION OF ABSTRACT

SAR
18. NUMBER

OF PAGES

10 19a. NAME OF RESPONSIBLE PERSON unclassified 
relied upon for acquiring raw radar backscatter for subsequent offline processing using algorithms we have developed at Scripps. OceanWaveS (Konny Reichert, Associate PI of this grant) continues the development of improved algorithms for realtime processing of radar data for bulk wave conditions such as significant wave height (Hs) and the directional wave spectrum. Additionally, we developed and deployed drifting miniature wavebuoys that provide directional spectra for comparison to the Xband data. Moored meteorological buoys were also developed and deployed to measure sea surface meteorological data. All deployed sensors were validated against each other, and the other instruments deployed by the other groups in the experiment. Beyond generating phase resolved wave fields, the XBand data set provided the opportunity to measure wind speed and direction (Jochen Horstman, NURC), indentify ocean surface fronts, develop wave breaking detection software, develop ocean current surface and depth profile algorithms, provide initial conditions and downstream data to initialize and validate wave modeling software developed by the other science teams (Dick Yue, MIT).

The lessons learned and algorithm validation achieved during HiRes is currently being used for continuous ongoing data collection and processing on the R/V Revelle and R/V Melville, supporting world-wide X-Band radar data analysis for ongoing ONR programs. Current and future ONR experiments involving littoral/river mouth measurements, and real time ship motion prediction also have benefitted from the validation achieved during the HiRes experiment. The use of X-Band radar to determine ocean wave bulk parameters, phase resolved wave fields, sea surface breaking, wind retrieval, and surface/depth current profiles remains an active area of research.

\section{WORK COMPLETED}

Several milestones have been achieved as part of this program. These include:

1. Completed processing X-Band radar data with SIO phase resolved wave inversion software, in both upwind and downwind directions, from both R/P FLIP and R/V Sproul, for comparison to 78 separate ATM swaths. The best result giving a phase resolved wave field correlation of $\mathrm{R}=$ 0.89 .

2. Completed processing R/P FLIP X-Band radar data with SIO phased resolved wave inversion software, and compared to Naval Postgraduate School (NPS) moored Datawell buoy. Twenty minute phase resolved time series correlations of $\mathrm{R}=0.74$ were achieved.

3. Completed processing of SIO miniature wavebuoy data for Hs and directional wave spectra. Compared results to NPS moored Datawell buoy, producing Hs scatter plot with correlation of $\mathrm{R}=0.94$. Compared time series and directional spectra to NPS moored Datawell and NPS drifters with excellent results. Compared wavebuoy sea surface height time series to X-Band radar inversion data, correlations as high as $\mathrm{R}=0.78$ for a 20 minute time series.

4. Used X-Band radar image averaging, and wave inversion processing, to identify a surface front off of the R/V Sproul during HiRes.

5. Provided X-Band radar data, both FLIP and Sproul, to Jochen Horstman at NURC for use in wind retrieval algorithm development.

6. Completed processing of SIO MET buoy data for sea surface atmospheric conditions. Provided data to Jochen Horstman at NURC. 
7. Helped define "grand challenge" data set times. Processed X-Band radar data, both FLIP and Sproul with SIO phase resolved wave inversion software, and provided results to Dick Yue at MIT for initialization and downstream predictive comparison to the MIT modeling efforts.

8. Processed X-Band data with a new algorithm to determine ocean current depth profiles, following the work of Stewart and Joy (SIO).

9. Worked with OceanWaveS GmbH to validate their wave inversion software and high resolution current software.

10. Installed X-Band radars on R/V Revelle and R/V Melville (supported under a separate DURIP award) to support ongoing and future ONR missions and research. Currently assisting in processing of DYNAMO experiment data.

11. Used HiRes experience and lessons learned to extend X-Band phase resolved processing to the littoral/river mouth environment (RIVET DRI).

12. Used HiRes experience and lessons learned to develop an experiment plan for ONR Environmental Sensing and Motion Forecasting (ESMF) program.

\section{RESULTS - ATM COMPARISONS}

Our approach to processing radar backscatter has been to process the data in the spectral domain. Sequences of radar scans are processed and filtered with 3D FFT techniques to determine the signal content attributed to ocean waves. Critical to this filtering process is the identification of the portion of the radar backscatter signal that follows the ocean wave dispersion relationship. SIO has developed algorithms which are run in a non-realtime mode to process the radar data, with attention paid to determining the ocean currents upon which the waves are riding. An example of radar backscatter is shown in Figure 1, the backscatter subset and map of the phase resolved surface wave field determined by our processing routines are shown in Figure 2.

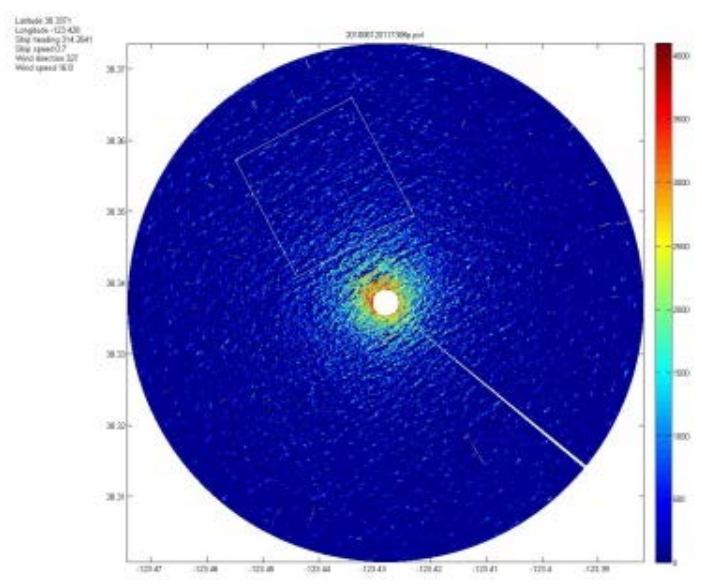

Figure 1. An example radar backscatter image. The white box is $2 \mathrm{~km} x 2 \mathrm{~km}$ and the color values shown represent 12 bit resolution of the $A / D$ programmed to sample the radar backscatter. 

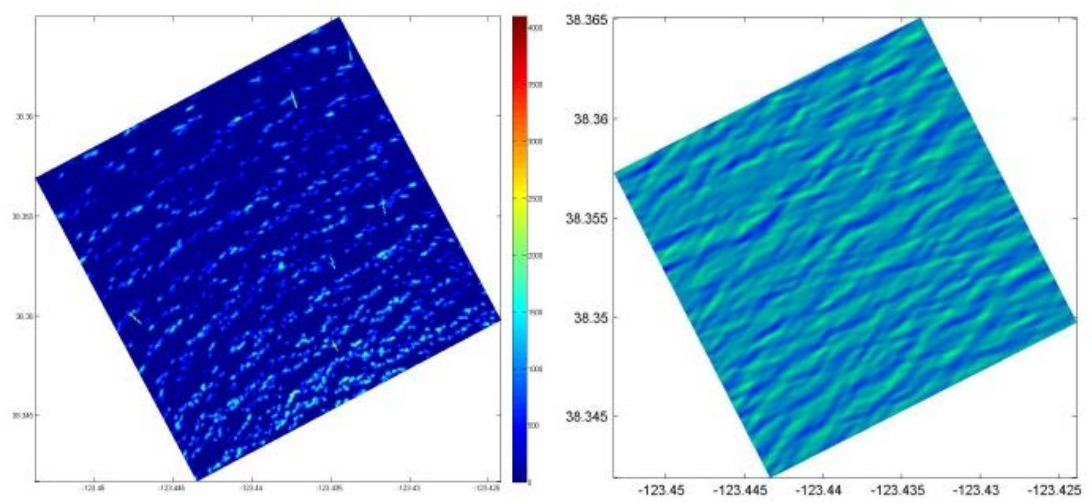

Figure 2. An example of the $2 \mathrm{~km}$ square processing domain, and the resultant phase resolved sea surface. Ocean wave heights are mapped to $-2 m$ to $2 m$ in elevation. The waves are propagating from top to bottom.

The phase resolved inversion process consists of a 3-dimensional FFT, spectral analysis to find the highest energy components along the shifted (by current) dispersion relationship, filtering, 3dimensional inverse FFT, and pixel-by-pixel scaling using an estimate of significant wave height (Hs) This Hs normalization can be driven by either the OceanWaveS WaMoS II bulk parameters or another sensor (bow-mounted altimeter or buoy). Figure 3 shows the 2-dimensional wavenumber (k) frequency (w) spectra.

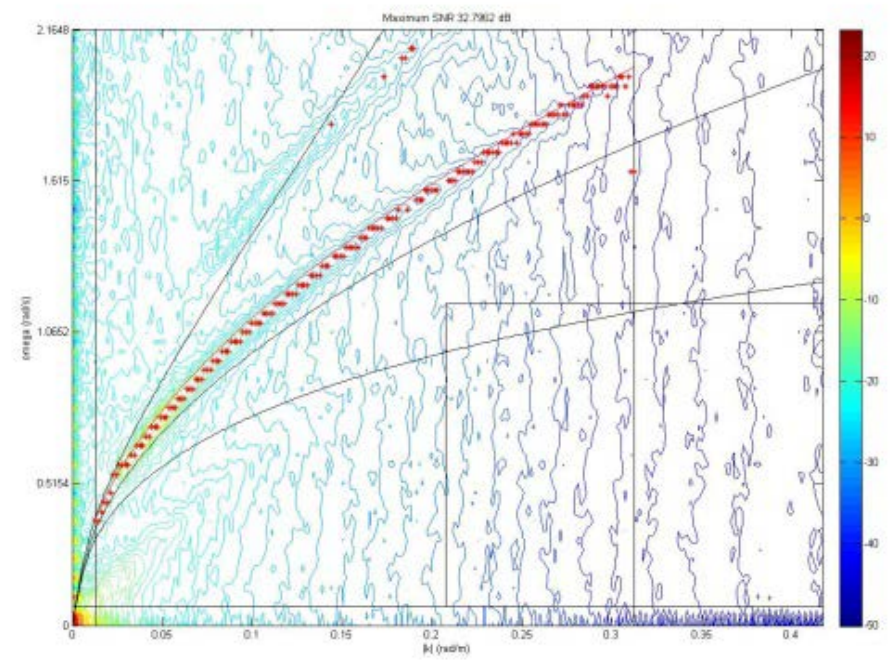

Figure 3. Example wavenumber-frequency spectrum showing the shifted dispersion relationship. The red asterisks indicate the (positively) Doppler shifted dispersion relationship energy band, indicating a current moving toward the radar (along with the waves). Also note the presence of the first harmonic of the shifted dispersion relationship, which is often present in large Hs situations due to wave shadowing.

Validation of the radar algorithms has been achieved using comparisons to independent measurements of the ocean wave field using airborne LIDAR and wave buoys. The two techniques are complementary as airborne LIDAR scans a swath of the sea-surface to provide a 'snapshot' of the sea surface only time aliased by the speed of the aircraft, while buoys can provide a point measurement of the waves as a function of time. Figure 4 shows a typical representation of our validation scheme. 


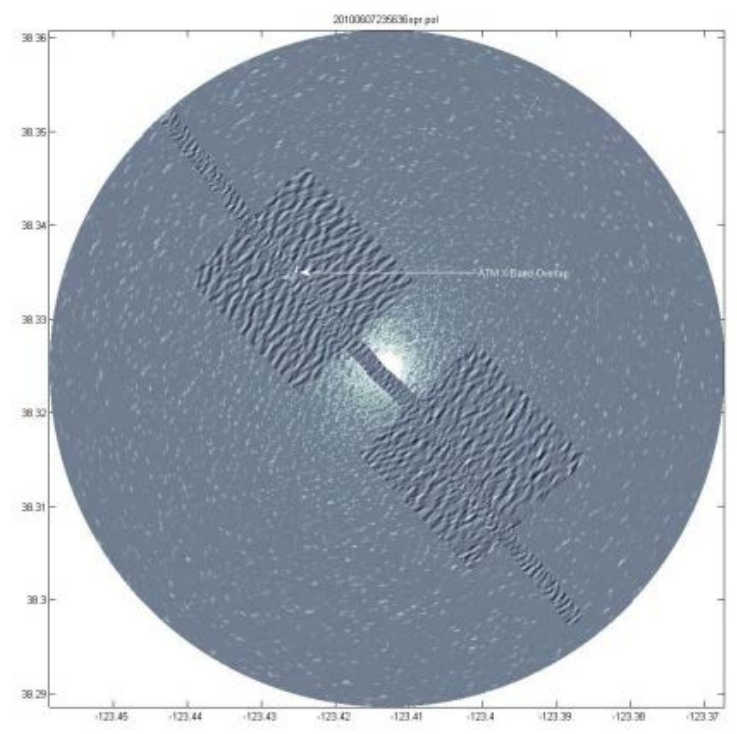

Figure 4. A backscatter frame from the $R / V$ Sproul $X$-Band radar, overlayed by two phase resolved wave fields (one upwind, one downwind), also overlayed with a swath of ATM data.

In order to do direct comparison to the ATM data, significant effort went into accurately georeferencing and time tagging the $\mathrm{x}$-band data. By taking known reference points, for example R/P FLIP, the radar data was registered spatially. Range and azimuth biases were removed for both radar systems. X-Band data acquisition was time registered to GPS pulse-per-second signals, which were embedded in the radar images. OceanWaveS and SIO worked together to develop this more accurate space/time registration process. To do ATM comparisons, small pieces of phase resolved wave fields had to be stitched together to generate a similar swath that the ATM produces in space and time.

Figure 5 is a zoom in of the overlap for a single radar scan.

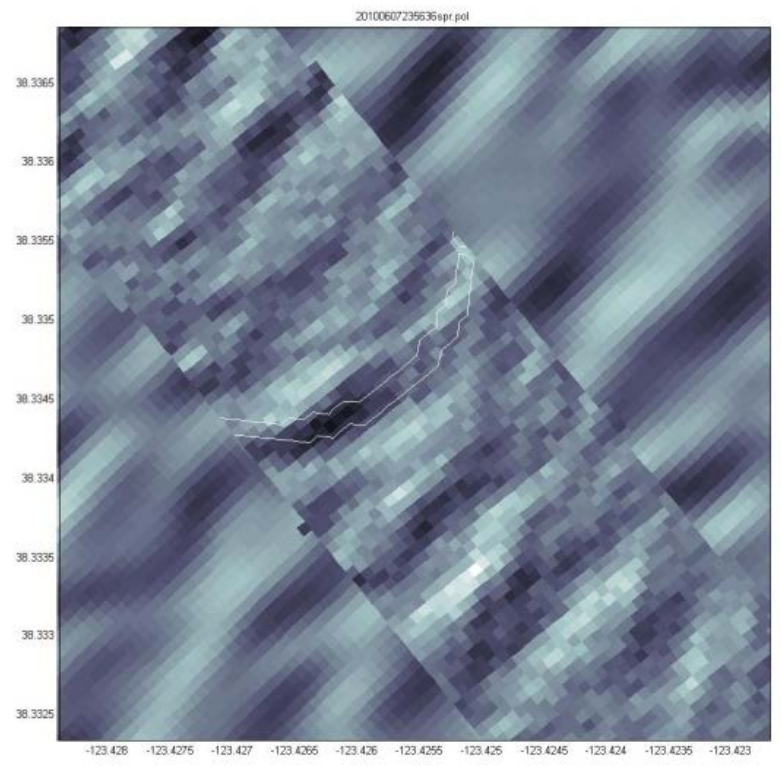

Figure 5. Zoom in showing the overlapping area in $(x, y, t)$ of the background inverted radar scan and overlayed ATM swath (white crescent). ATM data provided by W.K. Melville (SIO). 
Figure 6 shows the overall result of an up/down wind comparison between inverted X-Band radar scans and an ATM swath. Shown are the radar-derived wave heights mapped to the same time/spatial reference frame as represented by the LIDAR resolved sea surface.
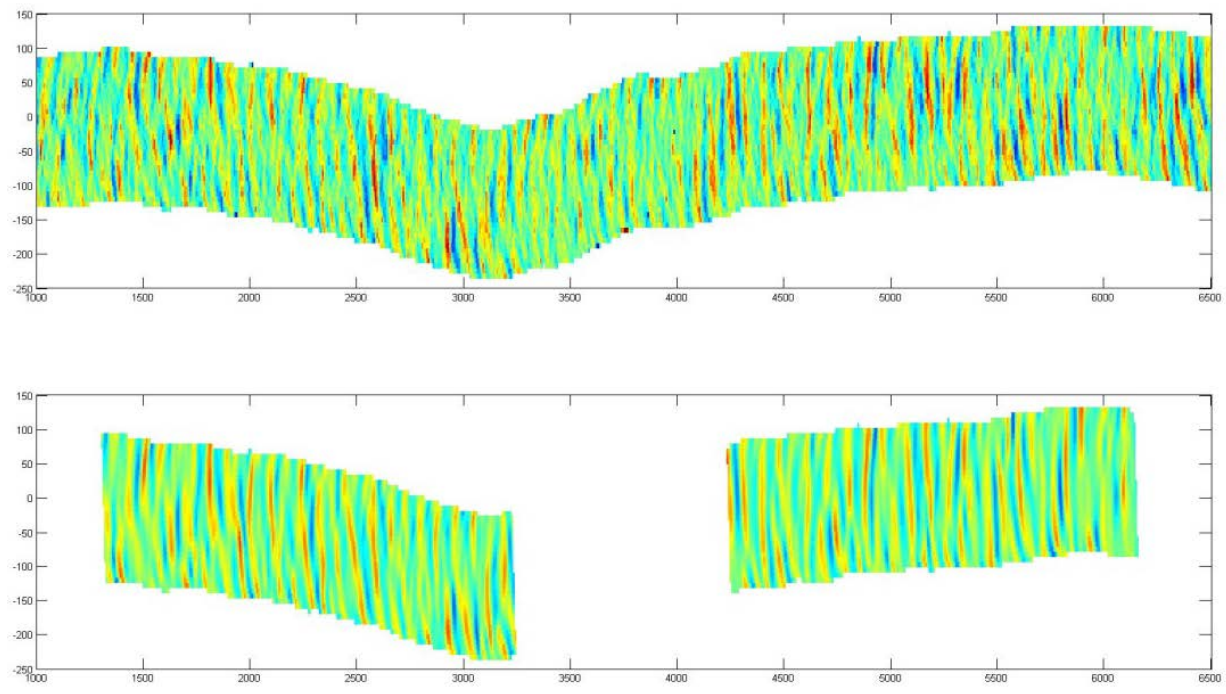

Figure 6. Top. LIDAR resolved ocean wave field provided by the airborne LIDAR system developed and operated by HIRES investigator Ken Melville. Along-flight distance is the horizontal axis and the swath width is the vertical. The aircraft has a speed of approximately $50 \mathrm{~m} / \mathrm{s}$. Bottom. The radar-derived wave field over the same space-time domain.

Seventy-eight separate ATM comparisons were done, with 2-dimensional spatial correlations reaching $\mathrm{R}=0.89$, with typical correlations of $0.75-0.80$. Figure 7 shows a 1 -dimensional spectral comparison between the radar inversion and the ATM. The ATM generates a more accurate spectrum at the lower and higher frequencies that are out of the X-Band inversion pass band.

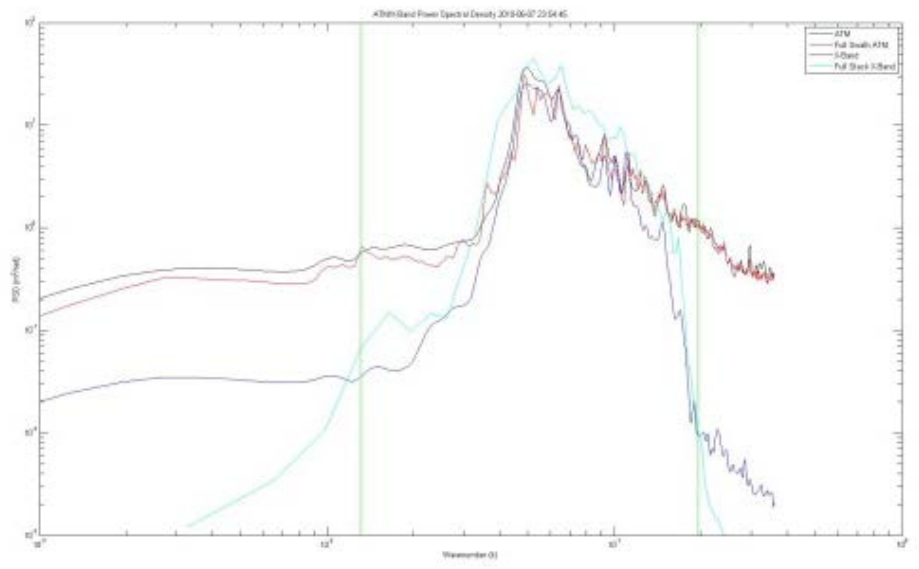

Figure 7. 1-dimensional wavenumber spectra of X-Band inversion vs. ATM, note the narrow band nature of the X-Band spectra due to the bandwidth of the shifted dispersion filtering process. 


\section{RESULTS - BUOY COMPARISONS}

In addition to the ATM data, wave buoy data was used to validate the X-Band wave inversion process. The NPS had a Datawell Mark III buoy moored in the field of view of the R/P FLIP X-Band radar. A single pixel of X-Band radar inversion data was used to generate a time series of sea surface heights at the location of the NPS buoy. Time series correlation of $\mathrm{R}=0.74$ was achieved over a 20 minute time period. Figure 8 shows this result.
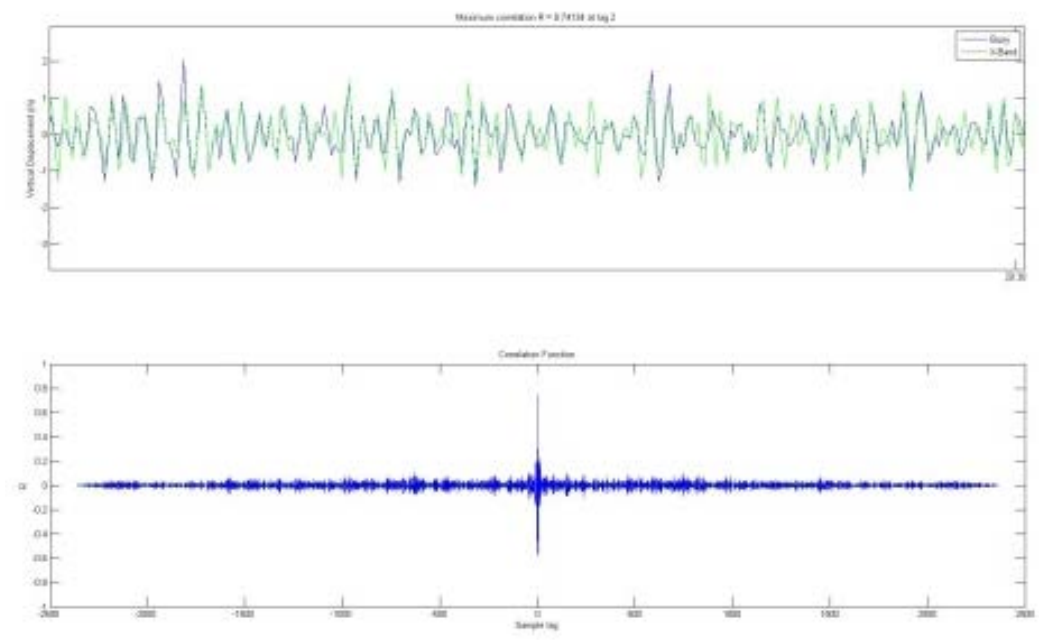

Figure 8. Top, X-Band single pixel vs. NPS Datawell buoy time series comparison. Bottom, correlation function illustrating the zero lag between the two observations.

SIO deployed its newly developed, GPS miniature drifting wave buoys, not only to validate their processing, but to use as yet another comparison to the X-Band wave inversions. Figure 9 (left) shows a directional spectrum comparison between one miniature wave buoy and the NPS Datawell. Given that the spectra compared favorably, we also compared Hs estimates between the two buoys. Figure 9 (right) shows a scatter plot comparing Hs estimates between the two buoys over all miniature wave buoy deployments, with a linear fit of $\mathrm{R}=0.94$.
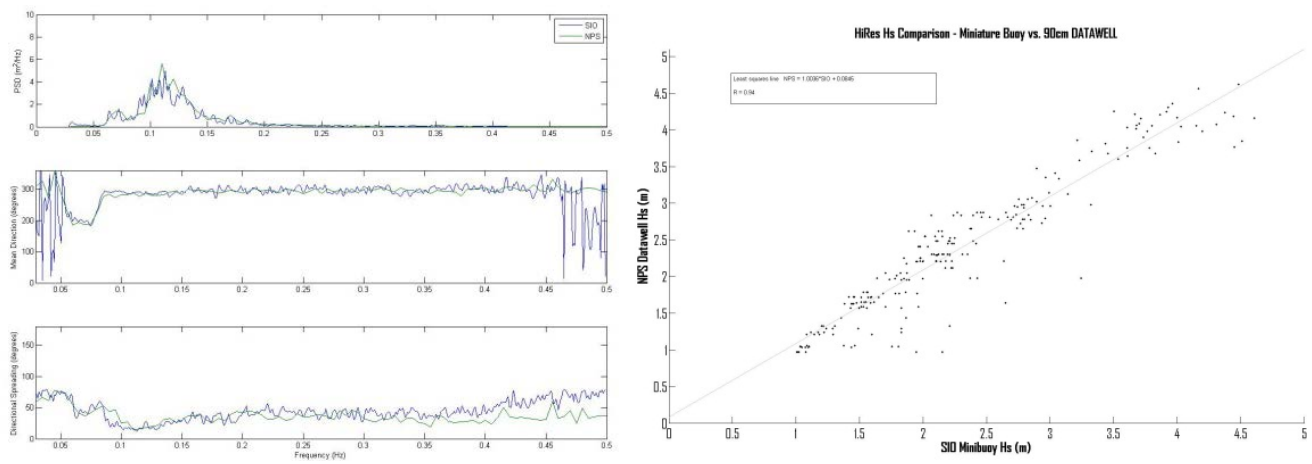

Figure 9. Left. Comparison of directional spectra between SIO miniature wave buoy (blue) and the moored NPS Datawell buoy (green). Right. Scatter plot of Hs values from SIO miniature drifting wave buoys and NPS Datawell buoy. 
As the miniature wave buoys drifted through the X-Band field of view, a pixel-by-pixel reconstruction of the wave buoy surface height was generated with the inverted radar data. Figure 11 shows the time series of the reconstruction.
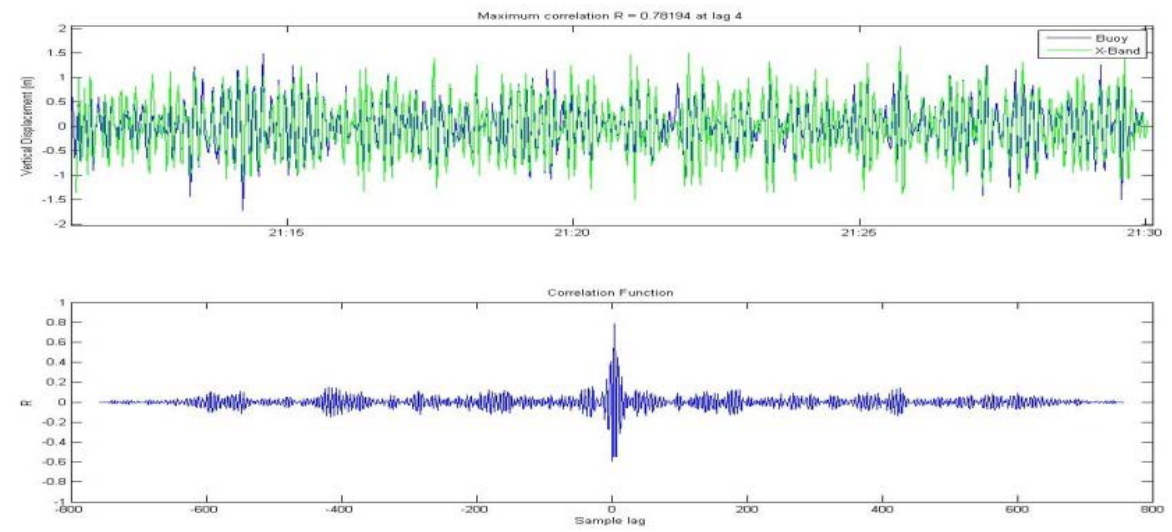

Figure 11. Top, comparison of $X-B$ and radar inversion pixel time series and drifting miniature wave buoy. Bottom, correlation function with $R=0.78$ over a 20 minute period. Zero time lag exists between the two observations.

\section{RESULTS - OCEAN FRONT DETECTION}

The R/V Sproul X-Band radar also proved useful in detecting surface fronts, using both averaged backscatter, and the wave inversion software. Figure 12 shows both results
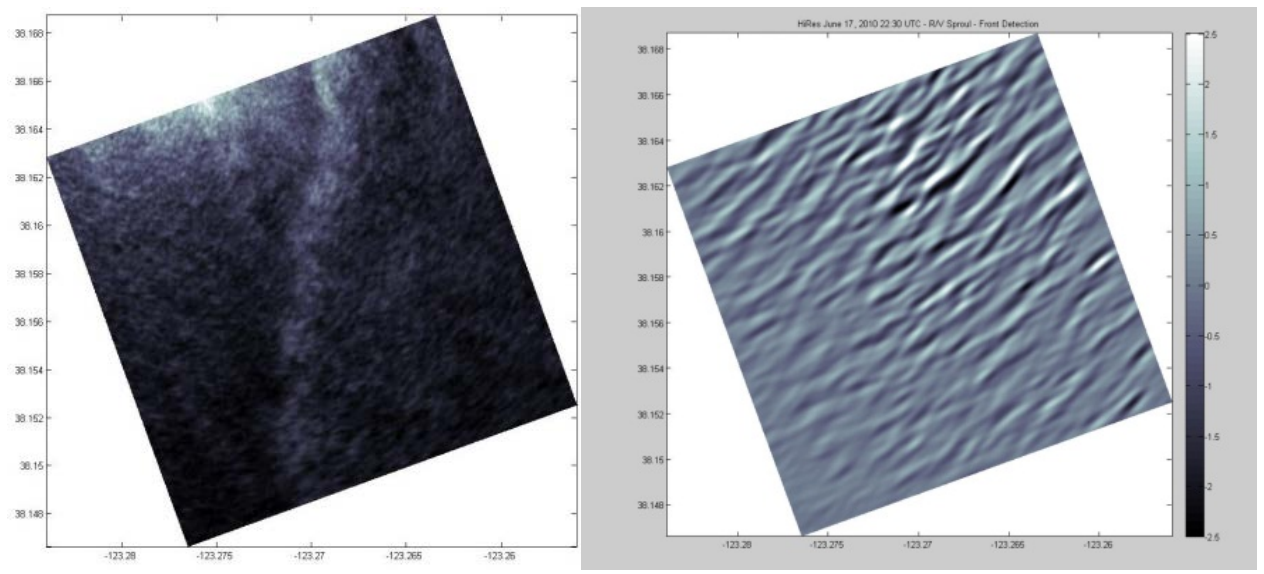

Figure 12. Left, 10 scan backscatter averages remove the varying wave field in the raw backscatter data, revealing a front on the ocean surface. Right, the inverted wave field also shows the difference in wave height, period, and direction across the surface front.

Recent application of this type of processing has proven valuable to the researchers who witnessed many severe weather fronts on the R/V Revelle in the middle of the Indian Ocean during the Dynamo cruise. 


\section{RESULTS - DECORRELATION SCALES}

By comparing the X-Band phase resolved wave inversions to their subsequent data, we find that ocean waves during the HiRes experiment loses correlation after approximately 5 peak periods. After that time period, very little phase relationship exists between the first frame of wave data and the last. Figure 13 shows the movement in both time and space of the correlation peak between the first frame of wave inversion data and the subsequent frames. The sawtooth pattern in the left part of Figure 16 exhibits the phase speed, and group speed, of the 14.5 second period waves. The steeper slope represents the phase speed, while the overall trend (slope) represents the group speed. The correlation "resets" at the wave period. On the right, one can see the correlation coefficient R decrease from unity to about 0.5 over roughly 5 periods.

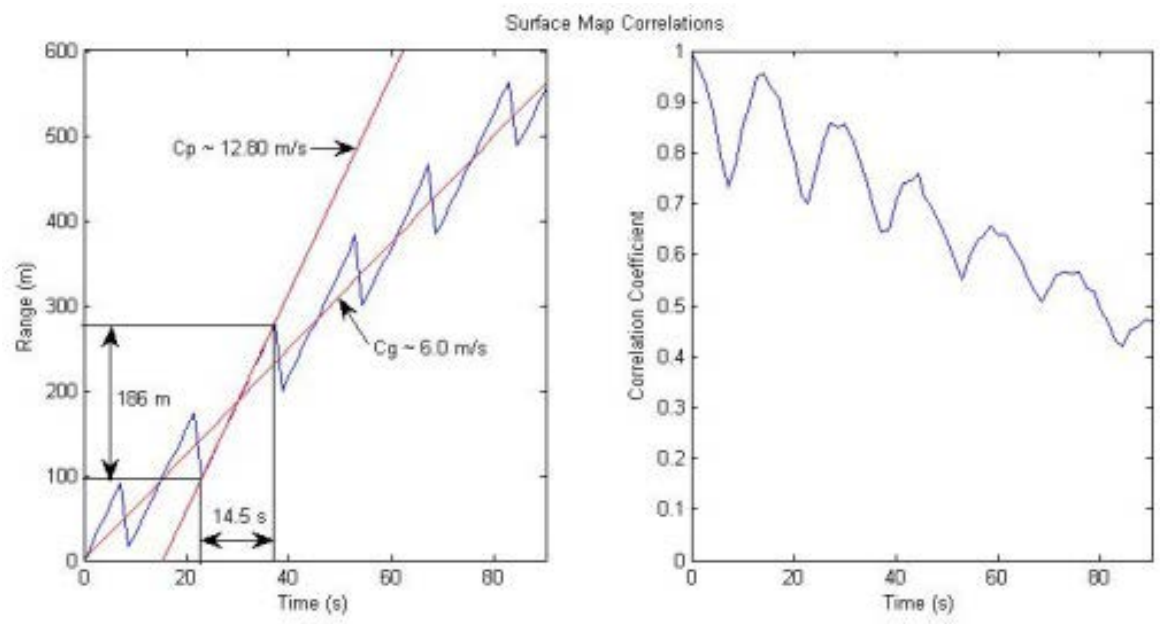

Figure 13. Time/space correlations of subsequent $X$-Band wave inversion frames.

\section{DATA DISTRIBUTION}

The X-Band radar data has proven useful to the other science parties involved in the HiRes experiment, as well as other non-HiRes groups.

- All the X-Band data, and SIO MET buoy data were sent to Jochen Horstmann of NURC for development of a wind retrieval algorithm (see JOATO publication).

- The whole group of HiRes scientists defined a "grand challenge" data subset, where high quality X-Band and ATM data were available as inputs and validation to the modeling efforts at MIT. The upwind wave inversions were used to initialize the data model, and the downwind wave inversions and ATM data were used to validate the model predictions. Due to the decorrelation scales previously discussed, validation beyond 5 peak periods proved to be difficult. Several different modelers supported by ONR have been provided wave data sets at the request of the program manager. 


\section{IMPACT/FUTURE APPLICATIONS}

The validation of the usefulness of X-Band radar data and phase resolved wave field processing has already had an impact on Naval research. Continuous data acquisition is ongoing on the R/V Revelle and R/V Melville. Near real-time X-Band radar bulk parameters, and miniature wave buoy directional spectra are actively being collected in different oceans around the world. Lessons learned from this program have supported the ONR Environmental Sensing Motion Forecasting (ESMF) Future Naval Capabilities (FNC) program, and two ONR Departement Research Initiatives (DYNAMO and RIVET). The ability to remote sense ocean waves from an at-sea craft has numerous naval applications including seakeeping for mobile offshore bases, ship-ship transfers of materials, helicopter operations, and maneuvering in high seastates. Lessons learned from the HiRes DRI will support efforts in Future Naval Capabilities (FNC) programs. The potential to generate deterministic descriptions of the ocean's surface at elevated sea states using both modern observational techniques and computationally intensive, nonlinear wave models now exists. Investments in research to successfully validate and improve these techniques and models will improve the planning and execution of at-sea naval operations and further our understanding of air-sea interaction processes during these conditions. The science X-band radar installations on the UNOLS vessels have also been accepted by the Department of State as a standard environmental sensor, allowing us to continuously collect wave data where the two ships operate. 